\title{
Scaling of the entropy change at the magnetoelastic transition in $\operatorname{Gd}_{5}\left(\mathbf{S i}_{x} \mathbf{G e}_{1-x}\right)_{4}$
}

\author{
Fèlix Casanova, Xavier Batlle,* and Amílcar Labarta \\ Departament de Física Fonamental, Universitat de Barcelona, Av. Diagonal 647, 08028-Barcelona, Catalonia, Spain
}

Jordi Marcos, Lluís Mañosa, and Antoni Planes

Departament d'Estructura i Constituents de la Matèria, Universitat de Barcelona, Av. Diagonal 647, 08028-Barcelona, Catalonia, Spain

(Received 5 September 2002; published 12 December 2002)

\begin{abstract}
Differential scanning calorimetry under a magnetic field $H$ has been used to measure the entropy change $\Delta S$ at the magnetoelastic transition in $\mathrm{Gd}_{5}\left(\mathrm{Si}_{x} \mathrm{Ge}_{1-x}\right)_{4}$ alloys, for $x \leqslant 0.5$. We show that $\Delta S$ scales with the transition temperature, $T_{t}$, which is tuned by $x$ and $H$, from 70 to $310 \mathrm{~K}$. Such a scaling demonstrates that $T_{t}$ is the relevant parameter in determining the giant magnetocaloric effect in these alloys, and proves that the magnetovolume effects due to $H$ are of the same nature as the volume effects caused by substitution.
\end{abstract}

DOI: 10.1103/PhysRevB.66.212402

PACS number(s): 75.30.Sg, 75.20.En, 75.40.Cx, 75.50.Cc

The magnetocaloric effect (MCE) has been studied for decades owing to its potential application to magnetic refrigerants. ${ }^{1}$ The MCE is the isothermal entropy change or the adiabatic temperature change arising from the application or removal of a magnetic field $H$ on a system with magnetic degrees of freedom. Many efforts have been devoted to the analysis of the MCE both in the vicinity of second-order magnetic phase transitions, where $\mathrm{Gd}$ is the element that shows the largest effect close to room temperature, ${ }^{1,2}$ and in order-disorder blocking processes, e.g., in molecular magnets. ${ }^{3}$ However, the MCE may be maximized in the vicinity of a first-order magnetoelastic phase transition, when the crystallographic transformation is field induced, resulting in an additional contribution to the entropy change $e^{4,1}$ : a giant MCE has been discovered in the $\mathrm{Gd}_{5}\left(\mathrm{Si}_{x} \mathrm{Ge}_{1-x}\right)_{4}$ compounds with $x \leqslant 0.5,{ }^{5-7}$ and recently in MnAs-based materials. ${ }^{8,9}$

This paper is aimed at studying the entropy change $\Delta S$ associated with the first-order magnetoelastic phase transition in $\mathrm{Gd}_{5}\left(\mathrm{Si}_{x} \mathrm{Ge}_{1-x}\right)_{4}$ alloys, which has lately aroused much discussion. ${ }^{5,10-13}$ Two compositional ranges are of interest. For $0.24 \leqslant x \leqslant 0.5$, the giant MCE is related to a firstorder magnetoelastic phase transition from a hightemperature paramagnetic (PM), monoclinic phase $\left(P 112_{1} / a\right)$ to a low-temperature ferromagnetic (FM), $\mathrm{Gd}_{5} \mathrm{Si}_{4}$-type orthorombic-I phase (Pnma), at temperatures ranging from $130 \mathrm{~K}(x=0.24)$ to $276 \mathrm{~K}(x=0.5){ }^{6,14}$ The structural transition occurs by a shear mechanism ${ }^{15}$ and yields a large volume contraction. The field-induced, reversible nature of the magnetostructural transition then results in strong magnetostriction ${ }^{14}$ and giant (negative) magnetoresistance. ${ }^{16}$ For $x \leqslant 0.2$, a second-order PM-toantiferromagnetic (AFM) transition occurs at $T_{N}$ (from $\sim 125 \mathrm{~K}$ for $x=0$ to $\sim 135 \mathrm{~K}$ for $x=0.2) .{ }^{6}$ Upon further cooling, a first-order AFM-FM transition takes place, whose temperature ranges linearly from about $20 \mathrm{~K}(x=0)$ to 120 $\mathrm{K}(x=0.2)$. MCE is related to such a first-order phase transition. The nature of the AFM phase is currently under discussion, ${ }^{17}$ and the magnetic structure may correspond to that of either a canted ferrimagnet, as proposed for $\mathrm{Nd}_{5} \mathrm{Ge}_{4}$ (Ref. 18) or a canted antiferromagnet, as for the Ge-rich region of the $\mathrm{Tb}_{5}\left(\mathrm{Si}_{x} \mathrm{Ge}_{1-x}\right)_{4}$ alloys. ${ }^{19,20}$ The AFM-FM tran- sition occurs simultaneously with a first-order structural transition from a high-temperature $\mathrm{Gd}_{5} \mathrm{Ge}_{4}$-type orthorombic-II phase to the low-temperature orthorombic-I phase, a large volume contraction also taking place. No structural anomalies are detected at the second-order PM-AFM transition. ${ }^{17}$ In the intermediate range $0.2<x<0.24$, orthorombic-II and monoclinic structures coexist. ${ }^{7}$

Differential scanning calorimetry (DSC) is the most suitable method to obtain the latent heat and entropy change at a first-order phase transition. ${ }^{21}$ In contrast, quasiadiabatic calorimetry, commonly used for the study of the MCE, ${ }^{22}$ is designed to measure the heat capacity $C_{p}$. However, at a firstorder phase transition, the experimental determination of $C_{p}$ is intrinsically uncertain due to the release of latent heat. ${ }^{23}$ DSC under $H$ is thus expected to be the ideal technique for the study of $\Delta S$ at first-order magnetoelastic transitions. We have developed a high-sensitivity DSC with built-in $H$. In this paper, the calorimetric measurement of $\Delta S$ as a function of $T$ and $H$ is reported for $\mathrm{Gd}_{5}\left(\mathrm{Si}_{x} \mathrm{Ge}_{1-x}\right)_{4}$ alloys. A $\Delta S$ scaling plot is obtained, where the scaling variable $T_{t}$ is the temperature of the first-order magnetoelastic phase transition. As $T_{t}$ is shifted with $x$ and $H$, the scaling of $\Delta S$ thus summarizes the giant MCE in the $\mathrm{Gd}_{5}\left(\mathrm{Si}_{x} \mathrm{Ge}_{1-x}\right)_{4}$ alloys. We also unambiguously show that calorimetric values of $\Delta S$ are in agreement with the indirect calculation obtained from the magnetization curves $M(H)$, using the ClausiusClapeyron equation. ${ }^{10,24}$

$\mathrm{Gd}_{5}\left(\mathrm{Si}_{x} \mathrm{Ge}_{1-x}\right)_{4}$ alloys with $x=0.1,0.18,0.2,0.25,0.3$, 0.365 , and 0.45 were prepared by arc melting admixtures of the pure elements in the desired stoichiometry under an argon atmosphere. As-cast buttons were cut into slices, and some were thermally treated for four hours at $950{ }^{\circ} \mathrm{C}$ under a $10^{-5}$-torr vacuum. The quality of the samples and their crystallographic structure were studied by room-temperature x-ray diffraction. ac susceptibility $(77-300 \mathrm{~K}, \quad \nu$ $=111-3330 \mathrm{~Hz}, H_{a c}=1.25 \mathrm{Oe}$ ) was used to check that the temperatures of the first- and second-order phase transitions were in agreement with values in the literature. ${ }^{6,7,17} M(H)$ curves were recorded up to $230 \mathrm{kOe}$ for $x=0.18$ and 0.45 , both in increasing and decreasing $H$, from 4.2 to $310 \mathrm{~K}$ with a temperature step of $3 \mathrm{~K}$. Calorimetric measurements were performed using two high-sensitivity differential scanning 


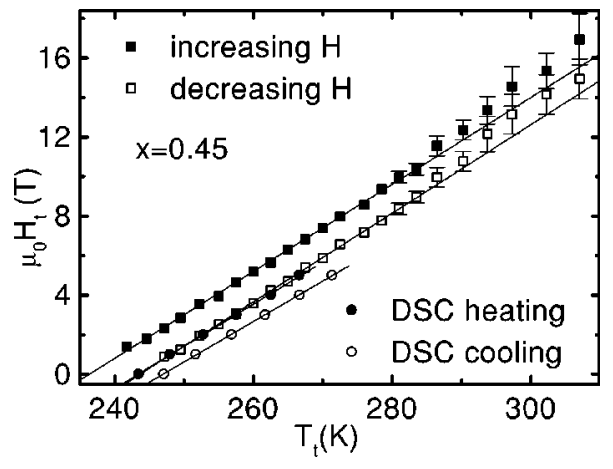

FIG. 1. Transition field $H_{t}$ plotted as a function of the transition temperature $T_{t}$ for $x=0.45$, obtained from $M(H)$ (increasing and decreasing $H$ ) and DSC data (cooling and heating). Solid lines are linear fits to experimental data.

calorimeters, specifically designed to study solid-solid phase transitions. Heating and cooling runs were performed within $77-350 \mathrm{~K}$ for $H=0$ in a $L \mathrm{~N}_{2}$ cryostat, and within $4.2-300 \mathrm{~K}$ under fields up to $50 \mathrm{kOe}$ in a $L \mathrm{He}$ cryostat. The calorimeter with built-in $H$ furnishes a sensitivity $\sim 10$ times larger at room temperature than that for a conventional DSC, while a reasonably high value is obtained at low $T$. Neither the thermometry nor the heat flow sensors are affected by $H$. Data do not depend on the cooling/heating rate. The upper limit of the operating range is $5 \mathrm{~K} / \mathrm{min}$. A detailed description will be published elsewhere. ${ }^{25}$

The measured $M(H)$ isotherms for $x=0.45$ were similar to those previously reported ${ }^{5,10}$ for $x=0.5$. The fieldinduced, first-order nature of the magnetoelastic PM-FM transition was evident from the data. It is worth noting that application of a field of $230 \mathrm{kOe}$ shifts $T_{t}$ by an amount of $\sim 80 \mathrm{~K}$. From the $M(H)$ curves a transition field $H_{t}$ is defined at each $T$ as the inflection point of the curve. The temperature dependence of $H_{t}$ is shown in Fig. 1, for measurements under increasing and decreasing $H$. A linear relation between $H_{t}$ and $T_{t}$ is obtained, which yields $d T_{t} / d\left(\mu_{0} H_{t}\right)$ $=4.5 \pm 0.2 \mathrm{~K} / \mathrm{T}$.

DSC data for $x=0.18$ (Fig. 2) also reveal the first-order nature of the low-temperature AFM-FM transition and the second-order nature of the high-temperature PM-AFM transition. The first-order transition shows (i) a large peak in $\dot{Q} / \dot{T} \equiv d Q / d T$, where $\dot{Q}$ is the recorded heat flow and $\dot{T}$ is the heating/cooling rate; (ii) a hysteresis of $2-3 \mathrm{~K}$ between cooling and heating; and (iii) a significant field dependence of $T_{t}$, which is estimated as the temperature at the maximum of the peak. The calorimetric data enable us to confirm the linear relation between $H_{t}$ and $T_{t}$, which is plotted in Fig. 1 for $x=0.45$, and yields $d T_{t} / d\left(\mu_{0} H_{t}\right)=4.8 \pm 0.1 \mathrm{~K} / \mathrm{T}$. A slight difference $(\sim 5 \mathrm{~K})$ in the determination of the zerofield transition temperature $T_{t}(H=0)$ from $M(H)$ and DSC curves is found, due to the use of different experimental devices. These values are in good agreement with those reported for $x=0.45, d T_{t} / d\left(\mu_{0} H_{t}\right)=4.5 \mathrm{~K} / \mathrm{T}$ (Ref. 14), and $x=0.43, d T_{t} / d\left(\mu_{0} H_{t}\right)=4.3 \mathrm{~K} / \mathrm{T} .{ }^{6} \mathrm{~A}$ linear $H_{t}\left(T_{t}\right)$ behavior is found for the other compositions, and the slopes are also

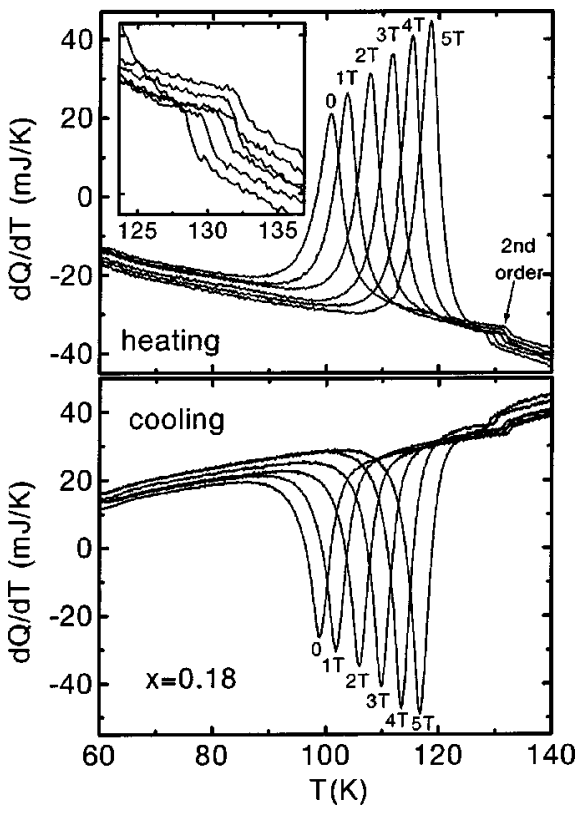

FIG. 2. DSC data for $x=0.18$ on heating and cooling the sample under $H$. Inset: details of the second-order transition on heating, from 0 (top) to $5 \mathrm{~T}$ (bottom).

consistent with published values. ${ }^{26,17}$ The second-order transition is observed in DSC as a small $\lambda$-type jump in the $d Q / d T$ baseline (Fig. 2, inset). Although DSC does not give the absolute value of $C_{p}$, the extrapolation at $T_{t}$ of the baselines at temperatures above and below the first-order transition provides a good estimation of $\Delta C_{p}$. It is found that $\Delta C_{p}$ is positive for the first-order AFM-FM transition for all compositions with $x \leqslant 0.2$ [see Fig. 3(a) for $x=0.1$ ], while a negative $\Delta C_{p}$ is obtained for the first-order PM-FM transition for $0.24 \leqslant x \leqslant 0.5$ [see Fig. 3(b) for $x=0.3$ ].

The absolute value of $\Delta S$ as a function of $T_{t}$ is shown in Fig. 4. As $T_{t}$ corresponds to the transition temperature of the first-order phase transition for each $x$ and $H$, this allows us to sweep $T_{t}$ from $\sim 70$ to $\sim 310 \mathrm{~K} . \Delta S$ was calculated as follows: (i) At $H=0\left(L \mathrm{~N}_{2}\right.$ cryostat) and up to $50 \mathrm{kOe}(L \mathrm{He}$ cryostat), by numerical integration of $(d Q / d T) / T$ throughout the first-order calorimetric peaks ${ }^{27}$; and (ii) indirect $\Delta S$ evaluation from the $M(H)$ isotherms up to 230 kOe for $x$ $=0.45$ and 0.18 , using the Clausius-Clapeyron equation at a

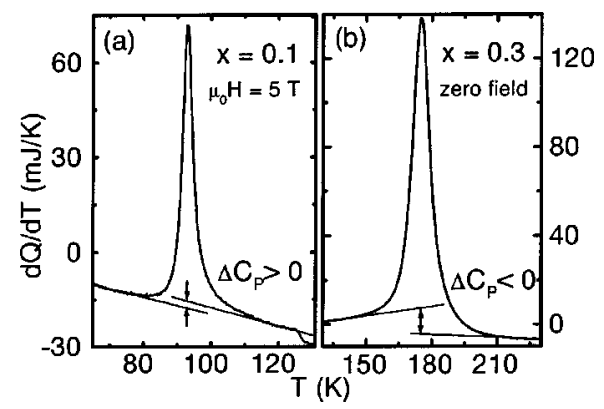

FIG. 3. DSC data for (a) $x=0.1$ on heating the sample with $\mu_{0} H=5 \mathrm{~T}$ and (b) $x=0.3$ on heating the sample without applied field. The opposite sign of $\Delta C_{p}$ for the two compositions is shown. 


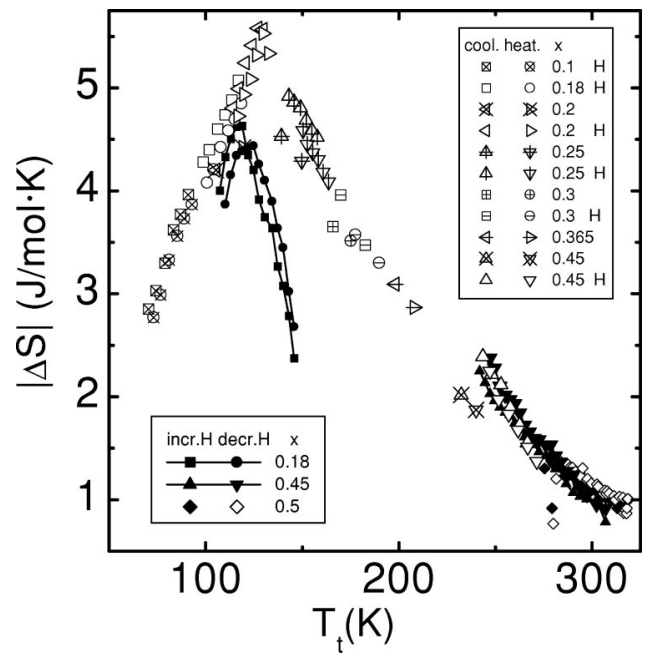

FIG. 4. Scaling of $|\Delta S|$ at the first-order transition for the $\mathrm{Gd}_{5}\left(\mathrm{Si}_{x} \mathrm{Ge}_{1-x}\right)_{4}$ alloys. A variety of applied fields and compositions are represented. Connected symbols correspond to values obtained from $M(H)$. Solid and open diamonds are from Ref. 10. Symbols labeled/not labeled with an $H$ correspond respectively to measurements with the $L \mathrm{He}$ (under $H) / L \mathrm{~N}_{2}(H=0)$ DSC.

constant pressure, $\Delta S=-\Delta M\left(d H_{t} / d T_{t}\right) .{ }^{24,10} \Delta M$ is determined from the magnetization jump at the transition. $\Delta S$ from the Clausius-Clapeyron equation reported by Giguère et al. for $x=0.5$, and obtained up to $70 \mathrm{kOe}$ (see Fig. 2 in Ref. 10), is also displayed in Fig. 4. As $T_{t}$ is tuned by both $x$ and $H,|\Delta S|$ values scale with $T_{t}$. This enables us to derive a scaling of $|\Delta S|$ for all $T_{t}$, i.e., for all compositions with $x \leqslant 0.5$. The values given in Ref. 10 also collapse onto this scaling plot. This shows that the relevant parameter in determining $|\Delta S|$ is $T_{t}$. In addition, the scaling is not a trivial consequence of the scaling of both $\Delta M$ and $d H_{t} / d T_{t}$, i.e., neither $\Delta M$ nor $d H_{t} / d T_{t}$ scale with $T_{t}$, which gives further relevance to the scaling of $|\Delta S|$. Notice also that $|\Delta S|$ extrapolates to zero at $T_{t}=0$, as expected from the third law of thermodynamics. The scaling is a consequence of the firstorder nature of the transition: at a constant $H$, the ClausiusClapeyron equation is written as $\Delta S=\Delta V\left(d P_{t} / d T_{t}\right)$, where $\Delta V$ stands for the volume jump and $P_{t}$ for the transition pressure. Therefore, $\Delta V$ and $\Delta M$ are related as $\Delta V / \Delta M=$ $-d H_{t} / d P_{t}$, and the scaling thus shows the equivalence of magnetovolume and substitution-related effects.

It is worth stressing that $\Delta S$ obtained from DSC measurements and from the Clausius-Clapeyron equation are coincident within the experimental error, for $x=0.45$ and 0.5 , and for $x=0.18$ in the temperature range where the AFM-FM transformation takes place. This shows that $\Delta S$ obtained from the Clausius-Clapeyron equation provides an excellent evaluation of the total entropy change at the first-order magnetoelastic transition. A comparision of $\Delta S$ to the entropy change obtained through the Maxwell relation ${ }^{10-13}$ was discussed elsewhere. ${ }^{28}$

Two diferent trends are shown in Fig. 4. For $0.24 \leqslant x$ $\leqslant 0.5,|\Delta S|$ associated with the PM-FM transition monotonically decreases with $T_{t}$, which is consistent with (i) $\Delta C_{p}$ $<0$ [Fig. 3 (b)], as expected from the thermodynamic rela- tion $d(\Delta S) / d T=\Delta C_{p} / T$; and (ii) $\Delta M$ decreasing monotonically with $T$. Moreover, negative $\Delta C_{p}$ may also be estimated from Ref. 26. In contrast, for $x \leqslant 0.2,|\Delta S|$ either decreases or increases, depending on $T_{t}$. Due to the magnetoelastic coupling, the application of $H$ shifts $T_{t}$, so that it is possible to observe both the AFM-FM transition at $T_{t}$ and, at high enough $H$, a PM-FM transition, when $T_{t}(H) \geqslant T_{N}$. The latter transition is still first-order due to the crystallographic transformation and arises from the PM-AFM transition. For the AFM-FM transition, $|\Delta S|$ increases monotonically with $T_{t}$, in agreement with $\Delta C_{p}>0$ [Fig. 3(a) and Ref. 26]. However, for the PM-FM transition, $|\Delta S|$ decreases with $T_{t}$ for $x$ $=0.18$ and $x=0.2$, in agreement with (i) $\Delta C_{p}<0$ [similar to Fig. 3(b)] and (ii) $\Delta M$ decreasing monotonically with $T$.

Consequently, $|\Delta S|$ is maximum for each composition at $T_{t}=T_{N}$, i.e., when, in the FM phase, the applied $H$ is large enough to shift the first-order transition to overlap to the second-order transition at $T_{N}$. Therefore, the largest value $|\Delta S|=5.58 \mathrm{~J} /(\mathrm{mol} \mathrm{K})$ occurs at $T_{t} \approx 131 \mathrm{~K}[\sim$ the highest value of $T_{N}$, which corresponds to $x=0.2$ (Ref. 6)]. All the foregoing suggests that $|\Delta S|$, and thus the MCE, will be maximum within the compositional range $0.2<x<0.24$, where the different crystallographic and magnetic phases coexist, and the two branches of $|\Delta S|$ join (Fig. 4). Finally, it seems that the slopes $d(|\Delta S|) / d T_{t}$ for the PM-FM transition for $x=0.18$ and that for $0.24 \leqslant x \leqslant 0.5$ are different. We argue that this is due to the high-temperature crystallographic phase being different (orthorombic-II and monoclinic phases, respectively, for $x=0.18$ and $0.24 \leqslant x \leqslant 0.5$ ).

In conclusion, DSC under $H$ has been used successfully to measure the entropy change at the first-order magnetoelastic phase transition for $\mathrm{Gd}_{5}\left(\mathrm{Si}_{x} \mathrm{Ge}_{1-x}\right)_{4}, x \leqslant 0.5$. We have shown that the transition entropy change scales with $T_{t}$ and it is in good agreement with the indirect measurements through the Clausius-Clapeyron equation. This is relevant for an understanding of the thermodynamics of first-order magnetoelastic transitions. The scaling of $\Delta S$ is a direct consequence of the fact that $T_{t}$ is tuned by $x$ and $H$ and it is thus expected to be universal for any material showing strong magnetoelastic effects, yielding a field-induced nature of the transition. $\Delta S$ is expected to (i) go to zero at zero temperature, (ii) tend asymptotically to zero at high temperature since the latent heat is finite, and (iii) display a maximum at that temperature for which both $\Delta M$ is maximized and $T_{t}$ shows the minimum field dependence. The specific shape of $\Delta S$ vs $T_{t}$ will depend on the details of the phase diagram, $T_{t}(x)$. Finally, the scaling of $\Delta S$ proves that the magnetovolume effects due to $H$ are of the same nature as the volume effects caused by substitution.

The financial support of the Spanish CICYT (MAT20000858 and MAT2001-3251) and Catalonian DURSI (2001SGR00066) are recognized. The Grenoble High Magnetic Field Laboratory, through the Improving Human Potential Program of the European Community, is acknowledged. F.C. and J.M. acknowledge DURSI for Ph.D. grants. 
*Electronic address: xavier@ffn.ub.es

${ }^{1}$ V.K. Pecharsky and K.A. Gschneidner, Jr., J. Magn. Magn. Mater. 200, 44 (1999).

${ }^{2}$ S.Y. Dan'kov, A.M. Tishin, V.K. Pecharsky, and K.A. Gschneidner, Jr., Phys. Rev. B 57, 3478 (1998).

${ }^{3}$ F. Torres, J.M. Hernández, X. Bohigas, and J. Tejada, Appl. Phys. Lett. 77, 3248 (2001).

${ }^{4}$ A. M. Tishin, in Handbook of Magnetic Materials, edited by K.H.J. Buschow (North-Holland, Amsterdam, 1999), vol. 12, pp. 395-524.

${ }^{5}$ V.K. Pecharsky and K.A. Gschneidner, Jr., Phys. Rev. Lett. 78, 4494 (1997).

${ }^{6}$ V.K. Pecharsky and K.A. Gschneidner, Jr., Appl. Phys. Lett. 70, 3299 (1997).

${ }^{7}$ V.K. Pecharsky and K.A. Gschneidner, Jr., J. Alloys Compd. 260, 98 (1997).

${ }^{8}$ H. Wada and Y. Tanabe, Appl. Phys. Lett. 79, 3302 (2001).

${ }^{9}$ O. Tegus, E. Brück, K.H.J. Buschow, and F.R. de Boer, Nature (London) 415, 450 (2002).

${ }^{10}$ A. Giguère, M. Földeàki, B. Ravi Gopal, R. Chahine, T.K. Bose, A. Frydman, and J.A. Barclay, Phys. Rev. Lett. 83, 2262 (1999).

${ }^{11}$ K.A. Gschneidner, Jr., V.K. Pecharsky, E. Brück, H.G.M. Duijn, and E. Levin, Phys. Rev. Lett. 85, 4190 (2000).

${ }^{12}$ J.R. Sun, F.X. Hu, and B.G. Shen, Phys. Rev. Lett. 85, 4191 (2000).

${ }^{13}$ M. Földeàki, R. Chahine, T.K. Bose, and J.A. Barclay, Phys. Rev. Lett. 85, 4192 (2000).

${ }^{14}$ L. Morellon, P.A. Algarabel, M.R. Ibarra, J. Blasco, B. GarcíaLanda, Z. Arnold, and F. Albertini, Phys. Rev. B 58, R14 721 (1998).
${ }^{15}$ W. Choe, V.K. Pecharsky, A.O. Pecharsky, K.A. Gschneidner, Jr., V.G. Young, Jr., and G.J. Miller, Phys. Rev. Lett. 84, 4617 (2000).

${ }^{16}$ L. Morellon, J. Stankiewicz, B. García-Landa, P.A. Algarabel, and M.R. Ibarra, Appl. Phys. Lett. 73, 3462 (1998).

${ }^{17}$ L. Morellon, J. Blasco, P.A. Algarabel, and M.R. Ibarra, Phys. Rev. B 62, 1022 (2000).

${ }^{18}$ P. Schobinger-Papamantellos and A. Niggli, J. Phys. Chem. Solids 42, 583 (1981).

${ }^{19}$ P. Schobinger-Papamantellos, J. Phys. Chem. Solids 39, 197 (1978).

${ }^{20}$ C. Ritter, L. Morellon, P.A. Algarabel, C. Magen, and M.R. Ibarra, Appl. Phys. Lett. 73, 3462 (2002).

${ }^{21}$ W. Hemminger and G. Höhne, Calorimetry. Fundamentals and Practice (Verlag Chemie, Weinheim, 1984).

${ }^{22}$ V.K. Pecharsky, J.O. Moorman, and K.A. Gschneidner, Jr., Rev. Sci. Instrum. 68, 4196 (1997).

${ }^{23}$ V.K. Pecharsky and K.A. Gschneidner, Jr., J. Appl. Phys. 86, 6315 (1999).

${ }^{24}$ A.J.P. Meyer and P. Tanglang, J. Phys. Radium 14, 82 (1953).

${ }^{25}$ J. Marcos, F. Casanova, X. Batlle, A. Labarta, A. Planes, and Ll. Mañosa (unpublished).

${ }^{26}$ V.K. Pecharsky and K.A. Gschneidner, Jr., Adv. Cryog. Eng. 43, 1729 (1998).

${ }^{27}$ This procedure gives reliable values for $\Delta S$ in first-order phase transitions. See, for instance, J. Ortín and A. Planes, Acta Metall. 36, 1873 (1988).

${ }^{28}$ F. Casanova, X. Batlle, A. Labarta, J. Marcos, Ll. Mañosa, and A. Planes, Phys. Rev. B 66, 100401(R) (2002). 